\title{
Metaobjetos de Aprendizagem
}

\author{
Alberto Bastos do Canto Filho, Engenharia Elétrica - UFRGS, alberto.canto@ufrgs.br \\ Liane Margarida Rockenbach Tarouco, PGIE/ UFRGS, liane@ penta.ufrgs.br \\ José Valdeni de Lima, PGIE/UFRGS, valdeni@ inf.ufrgs.br
}

\begin{abstract}
The competence to capacitation of human resources has not always received the ideal attention in engineering courses, creating a deficiency in the student's development, especially in areas like electronic, computation and electric, in which the product's creation goes by a training of the selling, producing and maintenance teams, in addition to the user himself. The current work introduces the concept of "Learning Metaobjects", a resource that has been investigated as an alternative insert in a methodology that looks to introduce the Learning Objects Project in engineering courses, breaking the cultural resistances to topics related to the human science, that frequently exist among the exact science's students.
\end{abstract}

Keywords: Learning Objects, Methodology, Project

\section{Resumo.}

A competência para a capacitação de recursos humanos nem sempre tem recebido a devida atenção nos cursos de engenharia, ocasionando uma deficiência formativa, especialmente em áreas como eletrônica, computação e elétrica, nas quais o desenvolvimento de produto passa pelo treinamento da equipes de vendas, produção, manutenção, e do próprio usuário. $\mathrm{O}$ presente trabalho introduz $\mathrm{o}$ conceito de 'Metaobjetos de Aprendizagem', um recurso que vem sendo investigado como alternativa inserida numa metodologia que visa introduzir o Projeto de Objetos de Aprendizagem em cursos de engenharia, quebrando as resistências culturais a temas relacionados às áreas de ciências humanas, freqüentemente existentes entre os estudantes de áreas de formação em ciências exatas.

Palavras-chave: Objetos de Aprendizagem, Metodologia, Projeto

\section{Introdução}

No ambiente de engenharia freqüentemente utiliza-se o termo treinamento para expressar os processos de ensino-aprendizagem associados ao ciclo de desenvolvimento de novos produtos. Especialmente em áreas como Eletrônica, Elétrica e Computação, qualquer novo produto desenvolvido deverá ser colocado em produção, vendido, ter assistência técnica, e ter assessoria ao cliente. Isto é, os profissionais de produção, manutenção, vendas, e os clientes devem ser "treinados". Os processos tradicionais de treinamento têm-se mostrado muito onerosos, especialmente para empresas multinacionais que, mesmo desenvolvendo seus produtos de forma centralizada, possuem fábricas, distribuidores, vendedores e clientes distribuídos geograficamente. Este tipo de necessidade, associado aos potenciais do uso de tecnologia em educação, tem levado grandes empresas a reverem suas estratégias de capacitação de pessoal. Citase como exemplo a CISCO, empresa provedora de soluções para Internet, que definiu estratégias para passar de cursos de treinamento tradicionais para o desenvolvimento de objetos disponíveis em bancos de dados, que possam ser reusados, pesquisados e modificados (CISCO, 1999), os RIOs - Reusable Information Objects. Com a inclusão 
de recursos tecnológicos nas etapas de treinamento, o ciclo de projeto já não pode ser considerado encerrado após as etapas de testes e documentação; o treinamento passa a ser tratado como uma etapa de projeto, que deve ser realizada a partir de especificações técnicas e restrições de prazo, custo e qualidade. Esta nova realidade pode representar um importante diferencial competitivo na área de engenharia, com novas oportunidades para empresas e profissionais. No entanto, a inclusão deste tema nos currículos de engenharia tem sido postergada. Deve-se isto não apenas à inércia natural dos processos de alteração curricular, mas também ao perfil do profissional de engenharia, freqüentemente voltado para ciências exatas e pouco motivado para as áreas de ciências humanas (PINTO, 2010). Agrava esta situação o fato do Projeto de Objetos de Aprendizagem ser uma área relativamente nova, onde as práticas e conceitos ainda não se encontram totalmente consolidadas.

Este trabalho propõe a utilização de Metaobjetos de Aprendizado inserida numa proposta de metodologia voltada para o desenvolvimento de competências para formação de recursos humanos dentro de cursos de engenharia. $\mathrm{Na}$ sessão 2, é apresentada a descrição da metodologia proposta; nas sessões 3 a 5 são apresentadas as premissas adotadas na metodologia; na sessão 6 são apresentados os resultados parciais do trabalho; na sessão 7 são apresentadas as conclusões.

\section{Metodologia}

$\mathrm{O}$ perfil do estudante de engenharia, voltado para ciências exatas e para o projeto e construção de dispositivos (engenhos), é caracterizado por um conjunto de motivações peculiares, impondo que os temas relacionados a ciências humanas sejam abordados de uma forma diferenciada. A Figura 1 mostra um cenário de ensino-aprendizagem dividido em quatro quadrantes, com a Engenharia situada nos quadrantes I e II (Conceitos e Aplicações de Ciências Exatas) e as áreas de Pedagogia e Psicologia situadas nos quadrantes III e IV (Conceitos e Aplicações de Ciências Humanas). Neste cenário, o Projeto de Objetos de Aprendizagem é apresentado como uma região de intersecção dos quatro quadrantes.

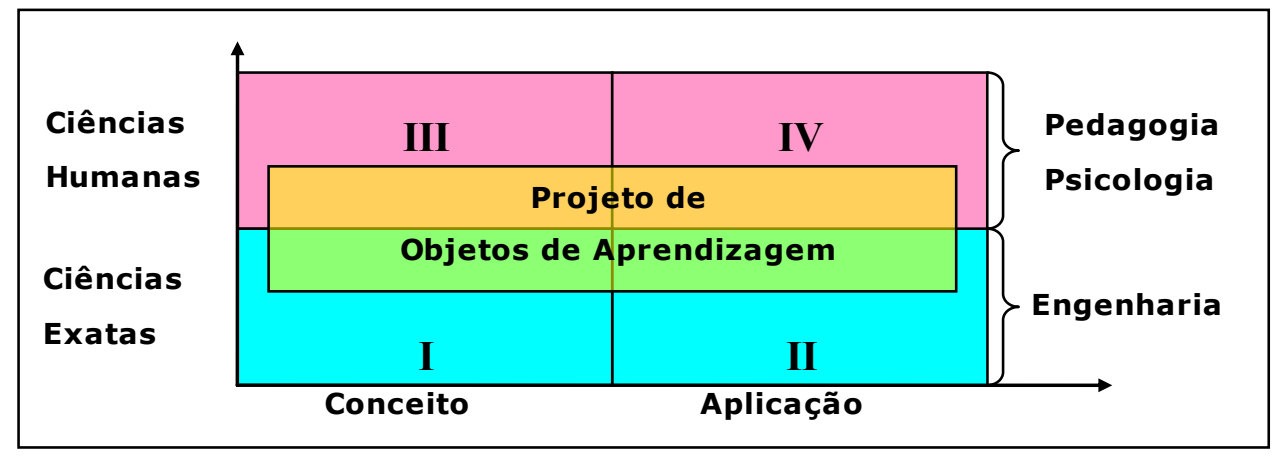

Figura 1 - Projeto de Objetos de Aprendizagem

A metodologia proposta neste trabalho aborda a capacitação de Recursos Humanos em Cursos de Engenharia através de Projeto de Objetos de Aprendizagem. O método considera três premissas: Introdução do tema a partir da Engenharia de Software, Uso de Metaobjetos de Aprendizagem, Conceituação Associada a Práticas de Projeto. As sessões a seguir apresentarão cada um destes fundamentos.

\section{Projeto de Objetos de Aprendizado a partir da Engenharia de Software}

A utilização de disciplinas de Engenharia de Software como ponto de partida para o Projeto de Objetos de Aprendizagem possibilita que os estudantes de engenharia 
iniciem seu aprendizado de Técnicas de Capacitação de Pessoal sem sair do ambiente de engenharia com o qual está acostumado. Trata-se de uma estratégia semelhante à utilizada por Daniela Reimann, que vem utilizando a moda para atrair estudantes do sexo feminino para a carreira de engenharia (REIMANN 2011). O aprendizado de Técnicas de Programação Orientada a Objetos e o uso de Técnicas de Modelagem de Sistemas proporciona um aprendizado de várias ferramentas e conceitos similares aos existentes no Projeto de Objetos de Aprendizagem, fornecendo para o estudante um conjunto de técnicas e ferramentas essenciais para o desenvolvimento de softwares educacionais. Uma das vantagens desta abordagem considera também que o Projeto de Objetos de Aprendizagem ainda não possui uma linguagem de engenharia própria, razão pela qual muitos autores vem utilizando a UML - Unified Modeling Language - como linguagem de projeto (CANTO et al, 2011). São exemplos desta prática, o Diagrama de Classes do modelo Netg apresentado por Katrien Verbert e Erik Duval (Figura 2.a), os modelos do "Learning Design Information Model" propostos pela IMS Global Learning Consortium (IMS 2003), ou o Diagrama de Atividades que descreve uma abordagem "Problem Based Oriented", parcialmente apresentado na Figura 2.b.

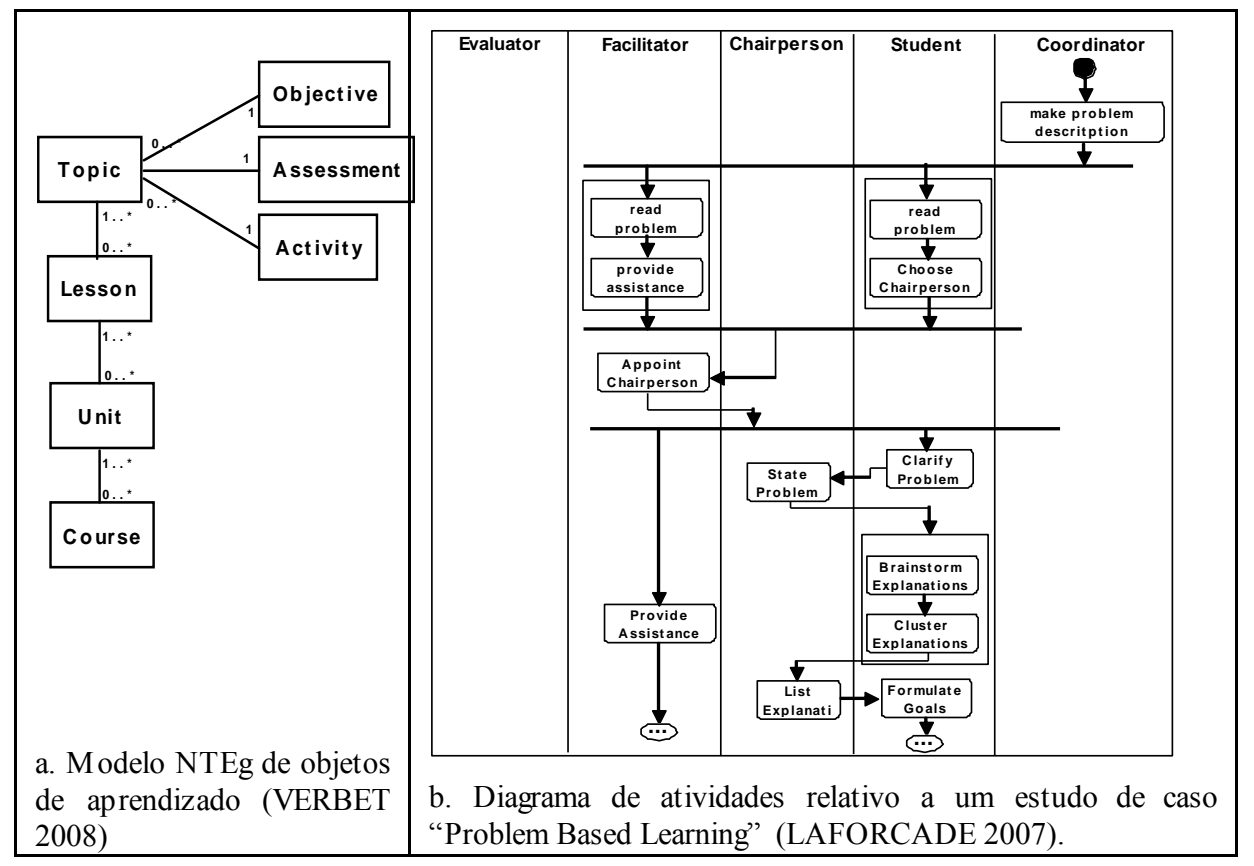

Figura 2. Exemplos de uso da UML

Não obstante as diversas similaridades, a passagem da Engenharia de Software para o Projeto de Objetos de Aprendizagem requer que sejam apresentados conceitos que ainda não estão completamente consolidados, razão pela qual se propõe uma clara conceituação de Objeto de Aprendizagem, Usabilidade, Uso e Reuso. As sessões a seguir apresentarão uma breve revisão bibliográfica e a conceituação adotada.

\subsection{Objeto de Aprendizage m}

Segundo o padrão IEEE 1484, Objeto de Aprendizagem é qualquer entidade digital ou não digital que possa ser utilizada para fins de aprendizado, educação ou treinamento (IEEE 2002). O caráter genérico da definição IEEE levou a críticas por parte de Johnson e Hall (2007), que a consideram exageradamente genérica, propondo sua própria definição: Objeto de Aprendizado é qualquer Recurso Digital de Aprendizado que possa ser Reutilizado. Apesar de mais específica, esta nova definição ainda é bastante ampla, pois abrange qualquer dispositivo construído com tecnologia digital, o que 
incluiria um relógio ou um computador de bordo de um avião, por exemplo. A definição de Meyer (2008) identifica com mais precisão os objetos abordados no contexto da metodologia proposta: Recurso de Aprendizado é um recurso digital utilizado para fins de e-learning. O quadro a seguir apresenta a adaptação da definição de Meyer à metodologia proposta.

Objeto de Aprendizagem é qualquer recurso digital utilizado nos processos de ensinoaprendizagem suportado por TICs (Tecnologias de Informação e Comunicação).

\subsection{Usabilidade}

Segundo a ISO 9241, usabilidade é a capacidade que um sistema interativo oferece a seu usuário, em um determinado contexto de operação, para a realização de tarefas, de maneira eficaz, eficiente e agradável. Observe na Figura 3, que a análise de requisitos de um sistema voltado para usabilidade passa, necessariamente, pela identificação dos usuários e suas tarefas.

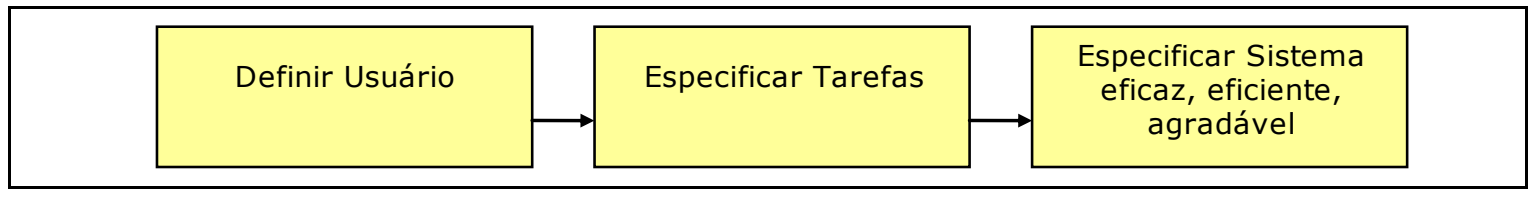

Figura 3 - Definição de um sistema visando a usabilidade

$\mathrm{Na}$ especificação de objetos de aprendizagem, identifica-se claramente o aprendiz (equipe a ser treinada) como usuário final, que possui como objetivo a aprendizagem. Adicionalmente, em processos de ensino-aprendizagem, pode-se identificar como usuário também o instrutor (equipe de desenvolvimento de produto), que compartilha o mesmo objetivo de aprendizagem eficaz, eficiente e agradável, por parte do aprendiz. No entanto o tipo de tarefa realizada pelo instrutor (desenvolvimento de objetos de aprendizagem) é substancialmente diferente do tipo de tarefa realizada pelo aprendiz. Isto é, para que o instrutor realize suas tarefas de forma eficaz, eficiente e agradável, os objetos de aprendizado devem possuir outros atributos, adicionais àqueles especificados para fins da usabilidade por parte do aprendiz. Para o instrutor, além da usabilidade instrucional, é necessário que os objetos de aprendizado sejam desenvolvidos de forma a facilitar a reutilização e proporcionar um ciclo de desenvolvimento mais eficiente (Figura 4). Decorre daí a caracterização do Reuso como uma das questões essenciais no Projeto de Objetos de Aprendizagem.

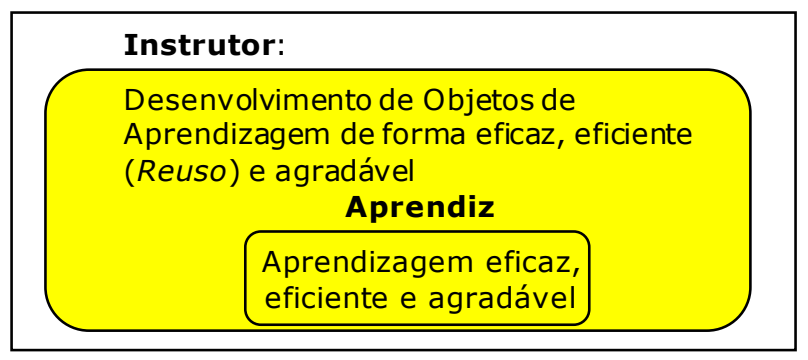

Figura 4 - Usabilidade de Objetos de Aprendizagem

\subsection{Uso e Reuso}

A idéia de reuso tem evoluído dentro de diversos setores de projeto, com variantes decorrentes da característica específica de cada área de conhecimento. Christopher Alexander el al (1997) comentam, sobre Padrões de Engenharia aplicados à construção 
de prédios e cidades: "Cada padrão de projeto descreve um problema que ocorre repetidas vezes no nosso ambiente, e descreve a essência da solução para este problema, de uma forma tal que ela possa ser utilizada um milhão de vezes sem nunca tê-lo feito da mesma forma". A incorporação do termo reuso como uma das questões essenciais da Engenharia de Software ocorreu no final dos anos 70 (FREDERIC, 1995), seguida por um período de amadurecimento turbulento, pautado pelas diferenças de metodologias e notações que levaram à chamada 'guerra dos objetos' (object war (Süß et al, 2004)). A necessidade de convergência terminou por prevalecer, levando três dos principais autores a unirem-se para a especificação da UML (Unified Modeling Language). De forma similar, o termo Reuso foi incorporado ao projeto de Objetos de Aprendizado a partir das práticas da Engenharia de Software, aplicado especialmente à reutilização de conteúdo, isto é, à reutilização do material entregue para o aprendiz, com o objetivo de suporte ao aprendizado (KOOHANG \& HARMAN, 2007). Embora exista um senso comum sobre o conceito de reuso de objetos de aprendizado, existem variantes conceituais. Por exemplo, Duval e Hodgins (2003), propõem o termo readequação (repurposing) para definir a utilização de um mesmo conteúdo para propósitos significativamente diferente daqueles para o quais os objetos foram originalmente concebidos. Esta definição, apesar de se enquadrar dentro do espírito proposto pela reutilização, pode dar margens a interpretações dúbias, ou até mesmo à criação de um paradoxo: se um objeto de aprendizado é criado com o objetivo original de proporcionar o aprendizado, o que significa 'utilização para propósitos diferentes daqueles para os quais ele foi originalmente concebido'? A conceituação proposta por Meyer (2008) mostra-se adequada para o bom entendimento do conceito de Reuso: Reuso de um Recurso de Aprendizado é qualquer tipo de uso de um recurso de aprendizado que já tenha sido utilizado num determinado contexto. Um recurso de aprendizado pode ser reutilizado sem qualquer modificação para fins de aprendizado ou de ensino; um recurso de aprendizado pode ser reutilizado em autoria por agregação; um recurso de aprendizado pode ser reutilizado em reautoria. Nesta definição a caracterização do Reuso como uma prática de autoria (criação) de objetos de aprendizado. Isto é, quem reusa os objetos de aprendizado é o Instrutor, e não o Aprendiz.

Tabela 1. Formas de reuso segundo diferentes autores

\begin{tabular}{|c|c|}
\hline Autor & Tipo de Uso / Reuso \\
\hline $\begin{array}{l}\text { Wiley (2009) apresenta } \\
\text { a proposta dos 4Rs: }\end{array}$ & $\begin{array}{l}\text { Reuse - utilizar exatamente na forma como se encontra } \\
\text { Revise - ajustar o objeto para que ele atenda a necessidades específicas } \\
\text { Remix - combinar (como estão ou modificados) diversos objetos } \\
\text { Redistribute - compartilhar o trabalho (Original), revisado ou remixado com outros }\end{array}$ \\
\hline $\begin{array}{l}\text { Meyer }(2008) \text { - } \\
\text { operações modulares } \\
\text { básicas, aplicadas sobre } \\
\text { recursos de } \\
\text { aprendizado: }\end{array}$ & $\begin{array}{l}\text { Modularização: divisão de um projeto (objeto de aprendizado) em módulos } \\
\text { Agregação: adição de novos módulos } \\
\text { Exclusão: remoção de módulos } \\
\text { Substituição: substituição de um módulo por outro } \\
\text { Reorganização: alteração da ordem dos módulos } \\
\text { Adaptação: transformação de um módulo }\end{array}$ \\
\hline $\begin{array}{l}\text { Duncan }(2009)- \\
\text { quantificação do reuso } \\
\text { de connections } \\
\text { modules }{ }^{1}\end{array}$ & $\begin{array}{l}\text { Uso: conta cada vez que o módulo é utilizado sem modificações } \\
\text { Reuso: "Uso menos um" } \\
\text { Tradução: contagem de todos os módulos derivados, com uma linguagem diferente da original } \\
\text { Modificação: contagem de todos os módulos derivados, na mesma linguagem do módulo } \\
\text { original } \\
\text { Reciclagem: soma de reuso, tradução e modificação } \\
\text { Todos Usos: soma de uso, tradução e modificação }\end{array}$ \\
\hline
\end{tabular}

${ }^{1}$ Connection modules: objetos de aprendizado disponíveis em repositórios, sob licença do tipo Creative Commons Attribution

V. $9 \mathrm{~N}^{\mathrm{o}}$ 2, dezembro, 2011 
A Tabela 1 apresenta formas de reutilização propostas por diversos autores. Observe a proposta de Duncan (2009) referente à contagem de módulos, na qual é caracterizada como Uso (por parte do Projetista/Instrutor) a primeira vez que o objeto (Connection Module) é utilizado. Cabe ressaltar que o Reuso no Projeto de Objetos de Aprendizagem freqüentemente é realizado através de técnicas de edição do tipo recortar, colar, inserir, aplicadas não apenas a softwares, mas também a imagens, áudio e textos. A reutilização típica do desenvolvimento de software (instanciamento e chamadas de subrotinas ou funções) também é uma prática de Reuso no projeto de objetos de aprendizagem.

\section{Metaobjetos de Aprendizagem}

A introdução do conceito de Metaobjeto de Aprendizagem (Quadro 1) tem por objetivo garantir que os fundamentos teóricos relacionados ao Projeto de Objetos de Aprendizado sejam abordados de forma a proporcionar aos estudantes a possiblidade de vivenciar a relação teoria-prática durante o processo de aprendizagem dos conceitos.

Metaobjetos de Aprendizagem são objetos de aprendizagem desenvolvidos com o objetivo de dar suporte ao ensino-aprendizagem de Objetos de Aprendizagem.

Quadro 1 - Definição de Metaobjeto de Aprendizagem

A característica recursiva de "utilização de objetos de aprendizagem para abordar objetos de aprendizagem" é similar ao uso de metadados, freqüentemente citados como "dados sobre dados" (Vicari et al, 2009). O uso de Metaobjetos de Aprendizagem na metodologia proposta parte do princípio que os conceitos abordados sejam fundamentos de técnicas de Projeto de Objetos de Aprendizagem e que, portanto, a apresentação deste conceito poderá ser feita com o uso da própria técnica fundamentada. Por exemplo, um Metaobjeto de Aprendizagem que tenha por objetivo a compreensão dos princípios de Mayer (2005) na produção multimídia, fará uso dos próprios princípios de Mayer (TAROUCO et al, 1999), proporcionando que o estudante vivencie uma experiência multimídia ao aprender os princípios e ferramentas de produção multimídia. Da mesma forma, um objeto de aprendizagem que utilize os eventos de Gagné (1987) para a aprendizagem dos eventos de Gagné. Ou uma apresentação sobre a ferramenta de autoria Wink (WINK, 2010) desenvolvida com o uso da própria ferramenta. O Quadro 2, a seguir, propõe um conjunto de princípios a serem observados no desenvolvimento de Metaobjetos de Aprendizagem.

1. Utilize Objetos de Aprendizagem para abordar Projetos de Objetos de Aprendizagem.

2. Para apresentar um conceito ou técnica de Projeto de Objetos de Aprendizado, aplique a técnica ou conceito apresentado.

3. Se não for possível aplicar os dois princípios acima, avalie se o conteúdo é realmente necessário.

4. Elabore a documentação de seus Objetos de Aprendizagem utilizando Objetos de Aprendizagem.

Quadro 2 - Projeto de Metaobjetos de Aprendizagem

Inúmeras pesquisas que fundamentam as técnicas de projeto de objetos de aprendizagem podem ser apresentadas através de Metaobjetos de Aprendizagem. A estratégia PTS (Picture plus Text plus Sound), pode ser utilizada para apresentar as 
investigações Riding e Cheema (1991) onde se identifica o perfil dos estudantes a partir de duas dimensões: Verbal/Imaginária e Global/Analítica, constatando que o uso de Áudio, Imagens ou Texto pode ser mais eficaz conforme o estilo cognitivo particular de cada estudante. Este conceito é reforçado pela Teoria da Carga Cognitiva e Sweeler, (2003), que considera a quantidade de informações que o ser humano pode processar a cada momento. Um outro exemplo é o uso de um título, contemplando a constatação das pesquisas de Douglas e Riding (1993), onde foi identificada uma maior eficácia de aprendizado quando é apresentado um título para estudantes com estilo cognitivo Global, apesar de apresentar pouco efeito para estudantes com estilo Analítico. A seguir, será apresentada a terceira premissa da metodologia, que complementa o uso de Metaobjetos de Aprendizado através da associação de Práticas de Projeto aos conceitos e técnicas abordados.

\section{Conceituação Associada a Práticas de Projeto}

O terceiro elemento da metodologia proposta é a adoção de uma abordagem de Aprendizagem Ativa. A profissão de engenheiro caracteriza-se pela realização de projetos exigindo, portanto, que os objetivos educacionais sejam estabelecidos dentro dos níveis superiores da taxonomia de Bloom (1956), de síntese e avaliação. O método desenvolvido propõe que o estudante utilize Melhores Práticas de Projeto de Objetos de Aprendizagem, associadas a técnicas de engenharia de software; isto é, na etapa de especificação de requisitos do projeto será necessário investigar conceitos de usabilidade, reusabilidade, e melhores práticas de ensino-aprendizagem, explorando os aspectos de psicologia educacional a partir de problemas de engenharia. O Quadro 3 mostra exemplos de boas práticas de usabilidade e reusabilidade, que podem ser exploradas como especificações técnicas complementares, no Projeto de Objetos de Aprendizagem.

- Projeto voltado para Usabilidade Instrucional: o projeto deve proporcionar o aprendizado de forma eficaz, eficiente e agradável, partindo de melhores práticas de utilização de multimídia, interatividade e e-learning;

- identificação de fragmentos: Lições de maior porte, devem ser fragmentadas em módulos menores reutilizáveis em outros contextos.

- integração de objetos: os objetos desenvolvidos devem ter a possibilidade de integração a outros;

- parametrização de textos, áudio e Imagens, de forma a facilitar a modificação do idioma, vocabulário, ou qualquer outra adaptação desejada. Por exemplo, num contexto de internacionalização, pode-se prever a facilidade de dublagem, uso de legendas, ou substituição de determinadas imagens por outras, com maior expressão numa determinada cultura;

- parametrização do "look and feel": os objetos devem ser desenvolvidos tendo a facilidade de adaptação a outros contextos que possuam identidade visual própria;

- tecnologia reutilizável: Quanto maior a flexibilidade da tecnologia utilizada no desenvolvimento, tanto maior serão as chances de reutilização do objeto;

- distribuição: a distribuição voltada para o reuso do Objeto de Aprendizagem; considera o tipo de licença de uso, a disponibilização de código fonte, o correto preenchimento dos metadados e a forma de distribuição propriamente dita;

- documentação: deve acompanhar o objeto de aprendizado um conjunto de documentos tais como manuais, tutores e exemplos, que facilitem o aprendizado da melhor forma de reutilização do objeto;

- padronização: embora ainda em fase de consolidação, diversos padrões voltados para o desenvolvimento de Objetos de Aprendizado (SCORM, IMS, IEEE, Dublin core, PBCore, etc.) estão disponíveis. A adoção de padrões de maior difusão no mercado irá ampliar as possibilidades de reuso.

Quadro 3. Exemplos de especificações complementares de Objetos de Aprendizagem 


\section{Resultados}

Por ocasião da redação deste trabalho a metodologia proposta estava parcialmente avaliada. A primeira etapa - Introdução do tema a partir da Engenharia de Software foi implementada através de um curso de extensão "C\# para Engenharia" ministrado nos meses de agosto / setembro de 2011. A Tabela 2 mostra o número de inscritos atividade de extensão comparado ao número médio de inscritos em atividades eletivas, oferecidas no segundo semestre de 2011 para o Curso de Engenharia Elétrica da Universidade Federal do Rio Grande do Sul, confirmando o interesse dos estudantes de engenharia pelo tema.

Tabela 2 - Comparação das Inscrições

\begin{tabular}{l|cc}
\hline & Vagas Ocupadas & \% Ocupadas / Oferecidas \\
\hline Extensão & 48 & $100 \%$ \\
\hline Eletivas & 14,9 & $53 \%$ \\
\hline
\end{tabular}

Como atividade complementar ao curso, foram desenvolvidos Metaobjetos de Aprendizagem sobre os seguintes temas:

- Eventos de Gagné;

- Instalação e Utilização do Wink;

- Ferramentas auxiliares para o uso de macros em Power Point.

\section{Conclusão}

Os Metaobjetos de Aprendizagem proporcionam aos estudantes a oportunideade de vivenciar uma aplicação do conceito simultaneamente à apresentação do próprio conceito, garantindo o atendimento de suas expectativas de visualização da aplicação prática dos conceitos. Paralelamente, a metodologia apresentada propõe que o estudante elabore projetos que contemplem os conceitos e técnicas apresentados, numa abordagem de Aprendizagem Baseada em Projetos que, ao mesmo tempo em que estabelece objetivos nos mais elevados patamares da taxonomia de Bloom (1956), traz o tema trabalhado para uma área de relevância no contexto de suas experiências e interesses preliminares (FELDER, 2005). Adicionalmente aos objetivos primários de desenvolvimento de competências para a capacitação de recursos humanos, a metodologia proposta permite que os melhores projetos dos alunos venham a integrar uma biblioteca de objetos de aprendizado voltados para ciências exatas, área carente de recursos pedagógicos de qualidade.

Por ocasião da elaboração deste trabalho, a avaliação de campo ainda encontrava-se em andamento. $\mathrm{O}$ número de estudantes inscritos na atividade de extensão "C\# para Engenharia" foi de 48, mais do que o triplo do número médio de alunos inscritos em disciplinas eletivas do Curso de Engenharia Elétrica, o que sinaliza a confirmação da hipótese de que as disciplinas de Engenharia de Software são um canal adequado para a introdução do projeto de objetos de aprendizado dentro do curso de engenharia investigado.

\section{Referências}

ALEXANDER, C. et al. A pattern language. Oxford University Press, New York, 1977. 
BLOOM, B.S. Taxonomy of educational objectives: the classification of educational goals: New York; Toronto: Longmans, Green. 1956. 207 p. Handbook I, cognitive domain.

CANTO, A. B. et al. UML para Modelagem de Objetos de Aprendizado. IX WORKSHOP TECNOLOGIA INFORMÁTICA APLICADA EM EDUCACIÓN Workshop Tecnología Informática aplicada en Educación (WTIAE), Buenos Aires, out 2011.

CISCO Systems, Reusable information object strategy- versão 3.0, 1999. Disponível em: $<$ http://www.cisco.com/warp/public/779/ibs/solutions/learning/whitepapers/el_cisco_rio.pdf $>$.

Acesso em 3/8/2011.

DOUGLAS, G.; RIDING R. J. The effect of pupil cognitive style and position of prose passage title on recall. Educational Psychology v. 13, n.3-4, p. 385-393, 1993

DUNCAN, S. M. Patterns of learning object reuse in the connexions repository, 2009. All Graduate Theses and Dissertations. Paper 423. Disponível em: $<$ http://digitalcommons.usu.edu/cgi/viewcontent.cgi?article=1398\&context=etd $>$. Acesso em 30/9/2011

DUVAL, E.; HODGINS, W. A LOM Research Agenda. In: WWW2003 - TWELF INTERNATIONAL WORLD WIDE WEB CONFERENCE, 20-24 May 2003, Budapest, Hungary. Proceedings... Disponível em: $<$ $\underline{\text { http://citeseerx.ist.psu.edu/viewdoc/download?doi }=10.1 .1 .86 .7978 \& \mathrm{rep}=\mathrm{rep} 1 \& \text { type }=\mathrm{pdf}>}>$. Acesso em: $10 / 10 / 2011$

FELDER, R. M.; BRENT, R. Understanding Student Differences. Journal of Engineering Education, n. 94, v. 1, p. 57-72, 2005. Disponível em: $<\underline{\text { http://citeseerx.ist.psu.edu/viewdoc/download?doi=10.1.1.167.8072\&rep }=\text { rep } 1 \& \text { type }=p d f>}$. Acesso em $5 / 10 / 2011$.

GAGNÉ, R. Instructional technology foundations. Hillsdale, NJ: Lawrence Erlbaum Assoc, 1987.

IEEE 1484.12.1-2002. Disponível em:

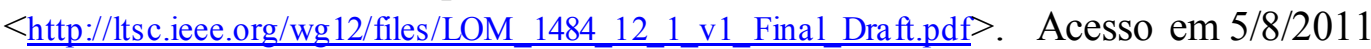

IMS Global Learnig Consortium. IMS Learning Design Information Model Version 1.0 Final Specification. 2003. Disponível em: $<$ http://www.ims global.org/learningdesign/ldv1p0/imsld infovlp0.html $>$. Acesso em 30/09/2011.

JONHSON, K.; HALL, T. Granularity, Reusability and Learning Objects. In: KOOHANG, Alex; HARMAN, Keith. Learning objects: theory, praxis, issues and trends. Santa Rosa, California: Informing Science Press, 2007.

LAFORCADE, P. Visualization of Learning Scenarios with UML4LD, Journal of Learning Design, v. 2, n. 2, p. 31-42, 2007.

MAYER, R. E. Introduction to Multimedia Learning. In: MAYER, R. E. (Ed.). The Cambridge Handbook of Multimedia Learning. New York: Cambridge University Press, p. 1-18, 2005.

MEYER, Marek. Modularization and Multi-Granula rity Reuse of Learning Resources. 2008.pp 19-23. Disponível em: $<\underline{\text { http://tuprints.ulb.tu- }}$ darmstadt.de/1156/1/Marek Meyer genehmigte Dissertation.pdf $>$. Acesso em 31/3/2011 
PINTO, G. P. P. R. Disciplinas Humanísticas na formação do engenheiro: fatores de resistência dos estudantes e estratégia educacional para a sua motivação. Tese apresentada ao programa de Pós-Graduação em Educação, Faculdade de Educação, Universidade Federal da Bahia, Salvador, 2010. Disponível em:

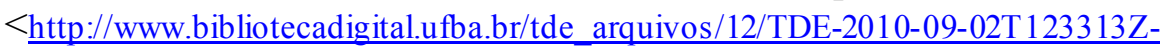
1857/Publico/Tese $\% 20-\% 20$ Gabriela $\% 20$ Pinto $\% 20$ Seg.pdf $>$. Acesso em 30/9/2011

REINMANN, D. Shaping Interactive Media with the Sewing Machine: Smart Textile as an Artistic Context to Engage Girls in Technology and Engineering Education.

International Journal of Art, Culture and Design Technologies (IJACDT), v. 1, n. 1, p. 12-21, 2011.

RIDING, R. J.; DOUGLAS, G. The effect of cognitive style and mode of presentation on learning performance. British Journal of Educational Psychology. v. 63, n. 2, p. 297-307, 1993.

RIDING R; CHEEMA I. Cognitive style - an overview and integration. Educational Psychology, v. 11, n. 3-4, p. 193-215, 1991.

Süß, J. G.; LEICHER, A.; CHABAREK, F. Software model engineering and reuse with the evolution and validation environment, GUELFI, N.; ASTESIANO, E; REGGIO, G. (Eds.): SCIENTIFIC ENGINEERING OF DISTRIBUTED JAVA APPLICATIONS, THIRD INTERNATIONAL WORKSHOP, FIDJ, November 27-28, Proceedings... Berlin: LNCS 2952, Springer 2004, p. 196-105, Revised Papers.

SWELLER, J. Cognitive load theory. A special issue of educational psychologist. LEA, Inc, 2003.

TAROUCO, L. M. R. et al. Multimídia Interativa: Princípios e Ferramentas. RENOTE - Revista Novas Tecnologias na Educação. Porto Alegre: Centro Interdisciplinar de Novas Tecnologias na Educação (CINTED - UFRGS), v. 7 n. 1, 2009. Disponível em:

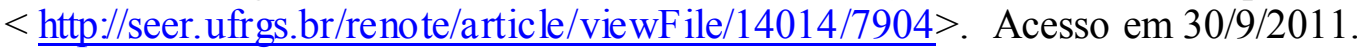

VERBET, K.; DUVAL, E. ALOCOM: A Generic Content Model for Learning Objects, International Journal on Digital Libraries, v. 9, n.1, p. 41-63, 2008

VICARI R M. et al. Proposta Brasileira de Metadados para Objetos de Aprendizagem Baseados em Agentes (OBAA). RENOTE - Revista Novas Tecnologias na Educação. Porto Alegre: Centro Interdisciplinar de Novas Tecnologias na Educação (CINTED - UFRGS), V. 8 Nº 2, 2010. Disponível em: $<$ http $/ /$ seer.ufrgs.br/renote/article/download/15257/9015 >. Acesso em 30/9/2011.

WILEY D. Impediments to Learning Object Reuse and Openness as a Potential Solution. Revista Brasileira de Informática na Educação, v. 17, n. 3, 2009. Disponível em: <http://www.br-ie.org/pub/index.php/rbie/article/viewFile/1022/1016>. Acesso em: 30/9/2011

WINK. Wink - Tutorial and Presentation Creation Software. 2010. disponível em: $<$ http $/ /$ www.debugmode.com/wink/>. Acesso em 30/9/2011. 